\title{
Serum Interleukin 19 in Patients with Vitiligo
}

\author{
A.A.Ebrahim, A.I.Mustafa and B.A.Abd-Elghany \\ Dermatology,Venereology and Andrology Dept., Faculty of Medicine, Benha Univ., Benha, Egypt \\ E-Mail: B. Abd-Elghany @gmail.com
}

\begin{abstract}
The aim of the present study is to evaluate the serum level of IL-19 in patients with Vitiligo to assess its role in Vitiligo.The present study will include 50 patients suffering from Vitiligo(GroupA). In addition,30 apparently healthy individuals of matched age and sex will be chosen as a control group (Group B). All patients will be selected from the outpatient clinic of Dermatology and Andrology Department of Benha University Hospitals. Estimation of serum levels Serum IL-19 levels will be measured using an ELISA kits. Five milliliters of venous blood sample will be collected from every participant in the study under complete aseptic precautions in the plain test tubes without anticoagulant.After coagulation, samples will be centrifuged (at $1500 \mathrm{~g}$ for $15 \mathrm{~min}$ ). The separated serum will bealiquoted and stored at $-20^{\circ} \mathrm{C}$ for subsequent assay of IL-19. The collected data will be tabulated and statistically analyzed using Special statistical methodsThe aim of the present study is to evaluate the serum level of IL-19 in patients with Vitiligo to assess its role in Vitiligo pathogenesis and its relation with studied disease parameters.
\end{abstract}

\section{Introduction}

Vitiligois an acquired skin disorder characterized by patchy depigmentation of the skin [1] . Vitiligo affects approximately $0.5 \%$ to $2 \%$ of the population worldwide, and the prevalence appears to be equal between men and women [2]. The pathogenesis of vitiligo is still not fully understood and mounting evidences have suggested that it might be related to autoimmunity and oxidative stress [3] . Although neither life-threatening nor symptomatic, the effect of vitiligo can be cosmetically and psychologically devastating, resulting in low selfesteem, poor body image and other negaive effects [4] .Vitiligo is classified into two main tytpes [5]

There is no known cure for vitiligo. For those with light skin, sunscreen and makeup are all that is typically recommended [6]. Globally about $1 \%$ of people are affected by vitiligo [7]. Preliminary evidence suggests a possible association with eating gluten [8].

There are remarkable new insights into the mechanisms of segmental vitiligo. So far, several hypotheses for segmental vitiligo have been put forward, including (i) neuronal mechanisms, (ii) somatic mosaicism and (iii)microvascular skin homing, whether or not leading to an autoimmune destruction of melanocytes [9].

$$
\text { Vitiligo is sometimes }
$$

associationwith autoimmuneand inflammatory

diseases such as Hashimoto's thyroiditis, scleroderma, rheumatoid arthritis, type 1 diabetes mellitus, psoriasis, Addison's disease, pernicious anemia, alopecia areata, systemic lupus erythematosus, and celiac disease [11].

There are associations between polymorphisms of genes of the IL19 cluster and their receptors and vitiligo, indicative of the part of IL19 and its receptor gene IL20RB in disease pathogenesis [ 12].

The Interleukin (IL)-19 is a member of the socalled IL-10 family of cytokines [13]. Interleukin (IL)19 isexpressedby epithelial cells with pro-inflammatory stimulation [14].
A distinctive feature of IL-19 is their ability to amplify themselves, once they are activated in inflammatory process, they will continuously produce the cytokine [15].

\section{Patients and methods}

The present study will include 50 patients suffering from Vitiligo(GroupA). In addition,30 apparently healthy individuals of matched age and sex will be chosen as a control group (Group B).

All patients will be selected from the outpatient clinic of Dermatology and Andrology Department of Benha University Hospitals.

\section{Ethical consideration}

An informed consents will be obtained from all the participants. The study will be approved by the ethics committee on research involving human subjects of Benha faculty of Medicine.

Type of the study:Case-control study.

\section{Inclusion criteria}

Patient with Vitiligo and the diagnosis will be based on clinical findings and the severity of the lesions will be assessed .

\section{Exclusion criteria}

Inflammatory skin conditions, burns, intralesional steroid injections andabrasions

Other conditions with similar symptoms.

All patients will be subjected to the following:

A complete history will be taken from each patient.

Complete general examination including: Body mass index (BMI), waist circumference, systolic and diastolic blood pressure.

Dermatological clinical examination will be done.

Clinical details of all patients will be recorded.

\section{Laboratory investigations}

Estimation of serum levels Serum IL-19 levels will be measured using an ELISA kits. 


\section{Procedure}

\section{Blood samples}

Five milliliters of venous blood sample will be collected from every participant in the study under complete aseptic precautions in the plain test tubes without anticoagulant.

After coagulation, samples will be centrifuged (at $1500 \mathrm{~g}$ for $15 \mathrm{~min}$ ). The separated serum will bealiquoted and stored at $-20^{\circ} \mathrm{C}$ for subsequent assay of IL-19.

\section{Statistical analysis}

The collected data will be tabulated and statistically analyzed using Special statistical methods.

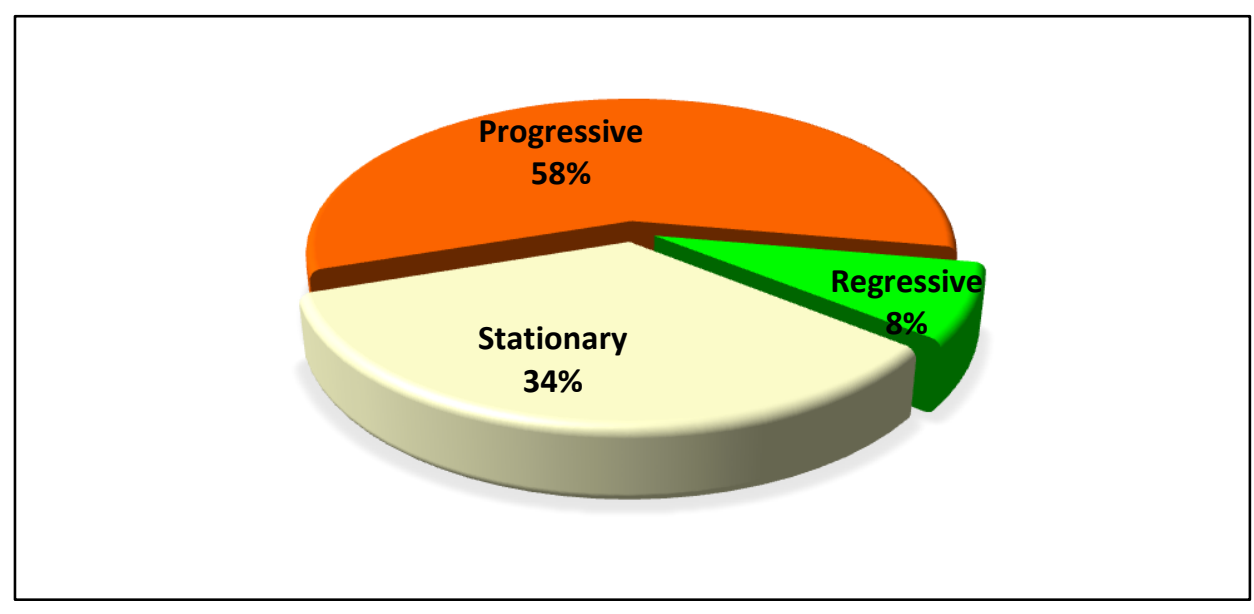

Fig (1) Course in all studied vitiligo cases.

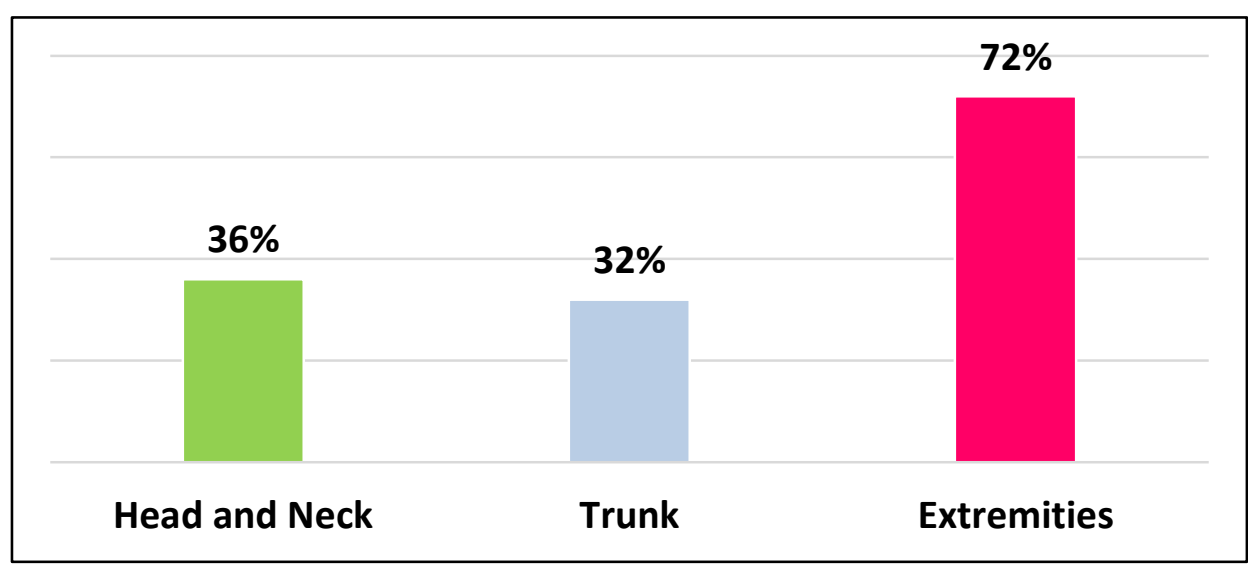

Fig (2) Site of affection in all studied vitiligo cases.

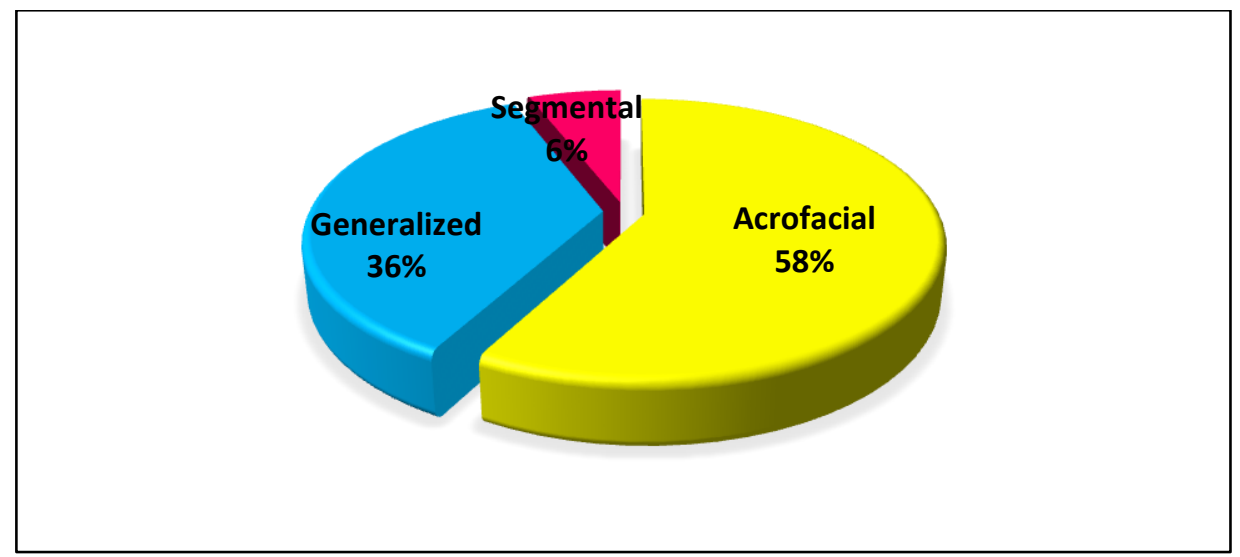

Fig (3) Clinical types in all studied vitiligo cases. 
Table (1) Validity of IL19 level for discrimination between vitiligo cases and control groups.

\begin{tabular}{ll}
\hline & IL19 \\
\hline AUC & 0.963 \\
Cut off & 27.1 \\
Sensitivity (\%) & 80 \\
Specificity (\%) & 93.3 \\
PPV (\%) & 95.2 \\
NPV (\%) & 73.7 \\
Accuracy (\%) & 85.0 \\
\hline
\end{tabular}

AUC, area under ROC, OC, receiver operating curve; PPV, positive predictive value; NPV, negative predictive value.

Receiver operating characteristic (ROC) curve of IL19 level was conducted for discrimination between vitiligo cases and control groups.
IL19 showed AUC of 0.963 . At best cut off value of 27.1 , sensitivity was $80 \%$, specificity was $93.3 \%$, PPV was $95.2 \%$, NPV was $73.7 \%$, and accuracy was $85 \%$.

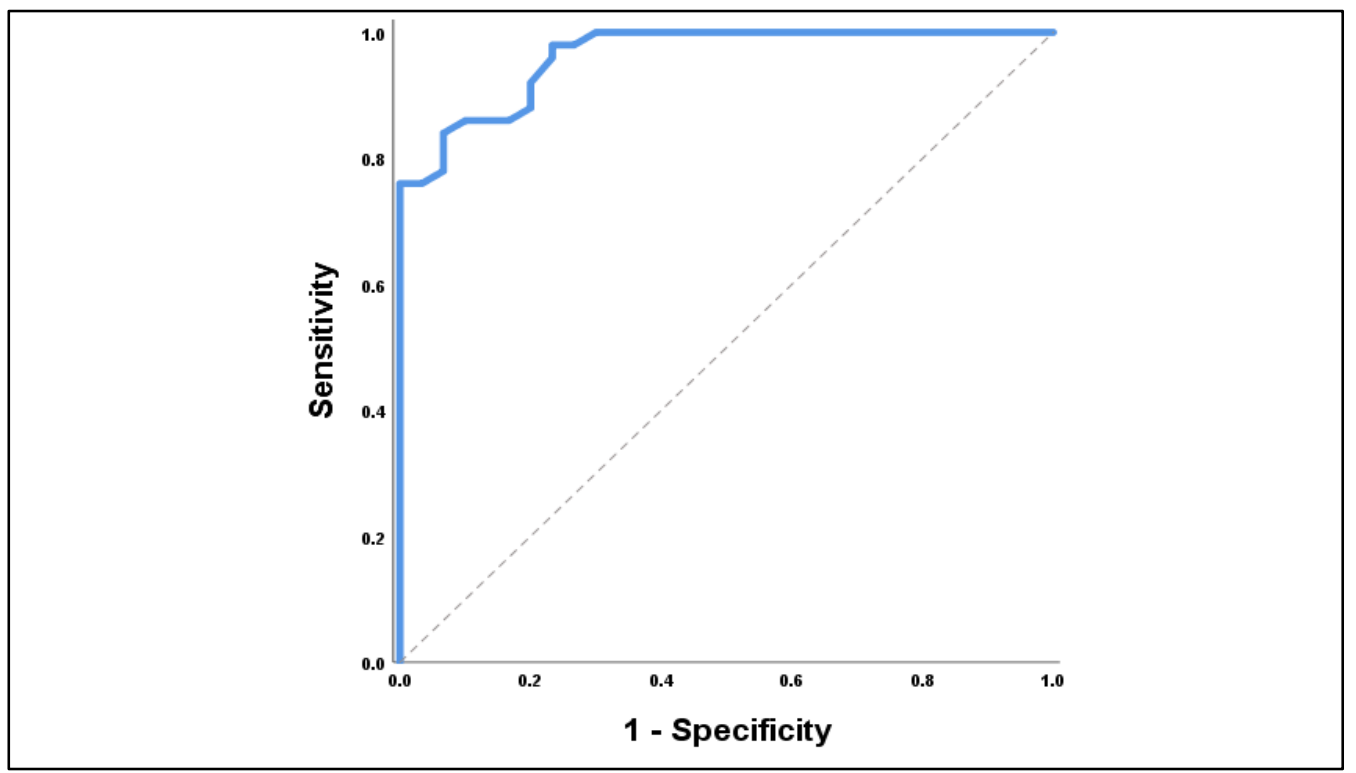

Fig (4) ROC curve of IL19 level for discrimination between vitiligo cases and control groups.

Table (2) Validity of IL19 level for detection of progressive disease.

\begin{tabular}{lc}
\hline & IL19 \\
\hline AUC & 0.832 \\
Cut off & 33.1 \\
Sensitivity (\%) & 93.1 \\
Specificity (\%) & 66.7 \\
PPV (\%) & 79.4 \\
NPV (\%) & 87.5 \\
Accuracy (\%) & 82 \\
\hline
\end{tabular}

AUC, area under ROC, OC, receiver operating curve; PPV, positive predictive value; NPV, negative predictive value.

ROC curve of IL19 level was conducted for detection of progressive disease.
IL19 showed AUC of 0.832 . At best cut off value of 33.1 , sensitivity was $93.1 \%$, specificity was $66.7 \%$, PPV was $79.4 \%$, NPV was $87.5 \%$, and accuracy was $82 \%$. 


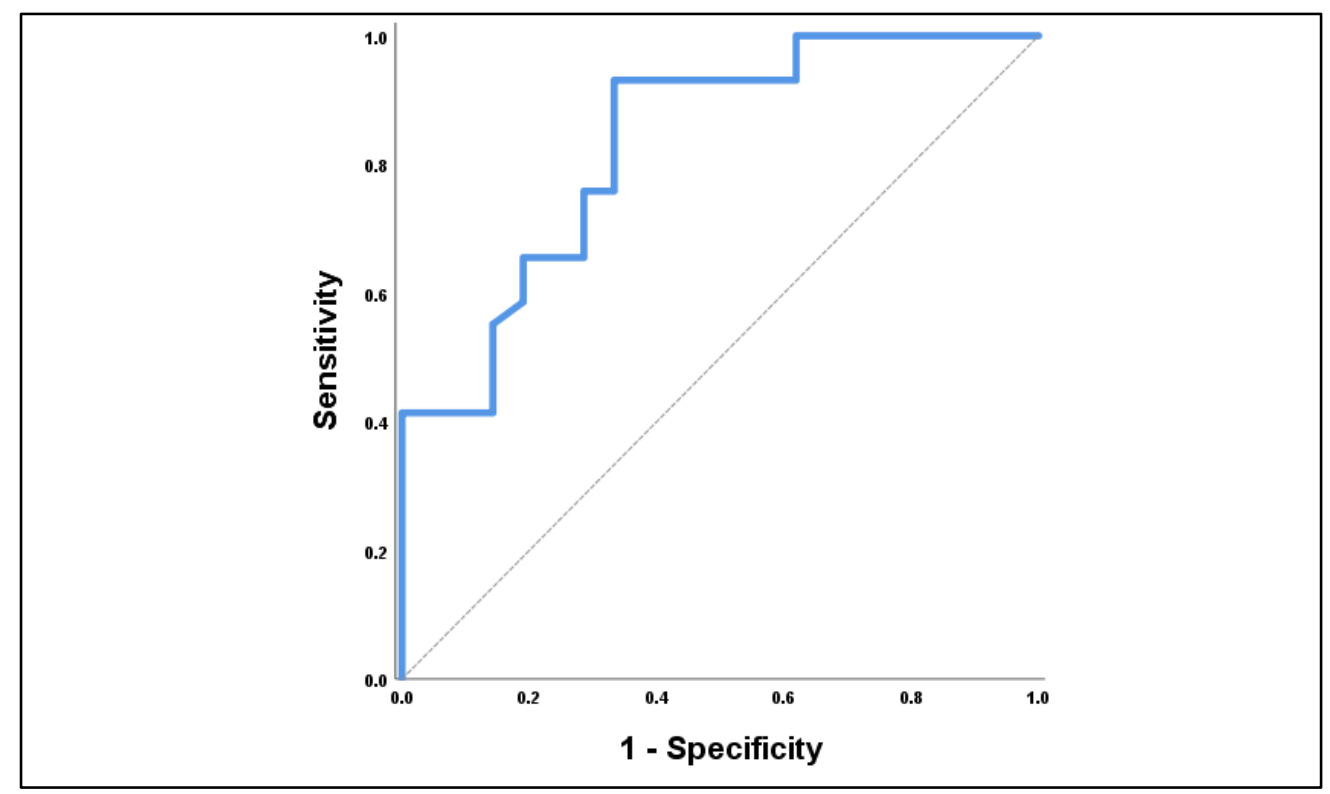

Fig (5) ROC curve of IL19 level for detection of progressive disease.

\section{Discussion}

Vitiligo is an acquired pigmentary disorder of unknownetiology, affecting approximately $0.1 \%-2 \%$ of the world population, without predilection for race or sex. It is characterized by white macules and patches, whose size increases over time, due to theloss of melanocytes. Vitiligo can appear at any time, and it significantly impairs the patients' quality-of-life [16].

Multiple pathogenetic factors have been proposed to clarify the etiology of vitiligo, including the neural theory, genetic predisposition, and impaired antioxidative defense [15].

In fact, growth factors produced by adjacentkeratinocytes regulate the proliferation and differentiation of melanocytes [17].

The mechanism of melanocyte loss in vitiligo is possibly related to keratinocyte-derived cytokines that stimulate melanocytes such as bFGF and SCF, or to those that inhibit melanocytes such as IL- 6 andTNF- $\alpha$. SCF is an essential factor for melanocyte survival [17].

Vitiligo affects approximately $1 \%$ of the world's population. Both adults and children are affected with no predilection for sex or ethnicity. The average age at onset lies around the second to the third decade of life [15].

Vitiligo is an acquired chronic depigmenting disorder of the skin overlying hair, and sometimes mucosa resulting from selective destruction of melanocytes, typical vitiligo lesions can be defined as milky white, non-scaly macules with distinct marginsand patches and that occurs secondary to selective destruction of melanocytes [16]. These lesions can appear in different shapes and sizes and may be present in any area of the tegument [17].

IL-19 gene contains 7 exons and is found on chromosome 1. Secreted IL-19 is made from 159 amino acids that create alpha-helix structure.

\section{Function [edit]}

The protein encoded by this gene is a cytokine that belongs to the IL-10 cytokine subfamily. This cytokine is found to be preferentially expressed in monocytes and less in B lymphocytes. The IL19 gene is expressed in resting monocytes and B cells. It is up-regulated in monocytes following stimulation with granulocyte-macrophage colony-stimulating factor (GM-CSF), lipopolysaccharide, or Pam3CSK4 [6].

\section{Immunoregulation function[edit]}

IL-19 increases the production of Th2 cytokines in $\mathrm{T}$-lymphocytes and induces expression of IL-10 in monocytes.

Disorder of the IL-19 production probably has an effect to different allergic reactions and other Th1 type athopic and skis pathogeneses, eg. psoriasis [7].

IL-19 also forms homologs with IL-20 and IL-24 and thus is able to bind the interleukin-20 receptor complex and lead to the activation of the signal transducer and activator of transcription 3 (STAT3). STAT3 is necessary for embryonal development and Th17 differentiation [8]. The IL-20 receptors are predominantly found in the mesenchymal region of tissues, lungs and reproduction organs.

A similar cytokine in mouse is reported to upregulate the expression of IL6 and TNF-alpha and induce apoptosis, which suggests a role of this cytokine in inflammatory responses. Alternatively spliced transcript variants encoding the distinct isoforms have been described [5].

\section{IL-10 family [edit]}

Interleukin-19 is a cytokine that belongs to the IL10 family of cytokines along with several other interleukins including IL-10, IL-20, IL-22, IL- 
24, IL-26, and several virus-encoded cytokines. It signals through the same cell surface receptor (IL-20R) that is used by IL-20 and IL-24.

\section{Summary}

Vitiligo is an acquired skin disorder characterized by patchy depigmentation of the skin(Yaghoobi R 2011). Vitiligo affects approximately $0.5 \%$ to $2 \%$ of the population worldwide, and the prevalence appears to be equal between men and women(Kyriakis KP 2009, and Allam M2013) .The pathogenesis of vitiligo is still not fully understood and mounting evidences have suggested that it might be related to autoimmunity and oxidative stress

The IL-19 gene contains 7 exons and is found on chromosome 1. Secreted IL-19 is made from 159 amino acids that create alpha-helix structure.

\section{Function[edit]}

The protein encoded by this gene is a cytokine that belongs to the IL-10 cytokine subfamily. This cytokine is found to be preferentially expressed in monocytes and less in B lymphocytes. The IL19 gene is expressed in resting monocytes and B cells. It is up-regulated in monocytes following stimulation with granulocyte-macrophage colony-stimulating factor (GM-CSF), lipopoly saccharide, or Pam3CSK4 [6].

The aim of this study was to evaluate the serum levels of IL-19 in patients with vitiligo and its correlation with disease severit

In this study, the serum IL-19 levels were examined in 50 patients with vitiligo compared to 30 genders and age matched healthy controls. Out of 50 patients with vitiligo 25 were males $(50 \%)$ and 25 (50\%) were females .

The result showed elevated serum IL-19 levels in patients with vitiligo, meanwhile, the elevated levels of IL-19 were positively correlated with duration of the disease and severity of the lesion.

We concluded that elevated serum IL-19 levels in patients with vitiligo suggested a potential involvement in the development and progression of vitiligo.

\section{Conclusion}

IL19 level was significantly higher in vitiligo cases when compared to control group, as well as in progressive disease, generalize type.

IL19 level was significantly correlated with extension.

Higher IL19 level was suggested to be independent predictor of vitiligo susceptibility, progressive and more extensive disease.

\section{Refranses}

[1] R.Yaghoobi, M. Omidian, N. Bagherani ()Vitiligo:areviewofthepublishedwok.J Dermatol
38: 419-1.isKP, PalamarasI,TseleE,MichailidesC,TerzoudiSCased etectionrates of vitiligo bygender and age.IntJ dermatol ,Vol. 48, PP.328-329.2011.

[2] AllamM,RiadH()Concisereview of recent studies in vitiligo .QatarMedJ, Vol.25(5),PP.1-19,2013.

[3] A. Alkhateeb, P.R. Fain, A. Thody, Epidemiology ofvitiligo and associated autoimmune diseases in Caucasian probands and their families .Pigment CellRes, Vol.16, PP.208-214,2013.

[4] S.K. Mattoo, S. Handa , I. Kaur, Psych-iatric morbidity invitiligo:prevalence and correlates in India .J Eur Acad DermatolVenereol, Vol.16,PP.573-578,2014.

[5] K. Ongenae , N. VanGeel, S. DeSchepper, Effect of vitiligo on self-reported health-related quality of Life.BrJDermatol,Vol. 152, PP.11651172,2013

[6] K. Ezzedine, V. Eleftheriadou, M. Whitton, "Vitiligo". Lancet, Vol. 386, PP.74-84.

[7] M. Whitton, M. Pinart, J.M. Batchelor, ("Evidence-based management of vitiligo: summary of a Cochrane systematic review". The British Journal of Dermatology, Vol.174 (5), PP. 962-9,2013.

[8] P.E. Grimes, R. Nashawati ,"The Role of Diet and Supplements in Vitiligo Management". Dermatol Clin (Review), Vol. 35 (2)PP. 235-243,2017.

[9] A. Taieb, F. Morice-Picard, T. Jouary, Segmental vitiligo as the bossible expression of cutaneous somatic,Vol.21,PP. 646-52,2008.

[10] F. Van Driessche, N. Silverberg ,"Current Management of Pediatric Vitiligo". Paediatr Drugs (Review), Vol.17 (4),PP.303,201513.

[11] K. Ezzedine , V. Eleftheriadou, M. Whitton, "Vitiligo".Lancet Segmental vitiligo as the bossible expression of cutaneous somatic, Vol.386,PP.74-84,2013.

[12] K. Kingo , E. Reimann, M. Karelson , a comprehensive overview Part I,Vol.221(3), PP.261-6,2010.

[13] G. Gallagher, H. Dickensheets, J. Eskdale, Cloning,expression and initial characterization of interleukin-19(IL-19), anovel homologue of human interleukin-10(IL-10) .GenesImmun, Vol.1, PP.442-50,2012

[14] .."Interleukin-19:Anewtarget to aim for?’Rheumatology,Vol.51(3), PP.399-400,2005.

[15] Alikhan, L.M. Felsten, M. Daly , Vitiligo: a comprehensive overview Part I.J Am Acad Dermatol, Vol. 65,PP.473-491,2011.

[16] K. Ezzedine, V. Eleftheriadou, M. Whitton Vitiligo. Lancet,Vol.386, PP.74-84,2015.

[17] R.G. Tarlé, L.M. Nascimento, M.T. Mira , Vitiligo - Part 1. An Bras Dermatol,Vol. 89(3),PP.461-470,2014 